\title{
A NEW PROOF OF UNIQUENESS FOR MULTIPLE TRIGONOMETRIC SERIES
}

\author{
J. MARSHALL ASH
}

(Communicated by R. Daniel Mauldin)

\begin{abstract}
Georg Cantor's 1870 theorem that an everywhere convergent to zero trigonometric series has all its coefficients equal to zero is given a new proof. The new proof uses the first formal integral of the series, while Cantor's proof used the second formal integral.
\end{abstract}

In 1870 Georg Cantor proved the following uniqueness theorem:

Theorem (Cantor [3]). If $\left\{a_{n}\right\}$ and $\left\{b_{n}\right\}$ are sequences of real numbers and if

$$
\frac{1}{2} a_{0}+\sum_{n=1}^{\infty} a_{n} \cos n x+b_{n} \sin n x
$$

converges at each $x$ to 0 , then the series vanishes identically; i.e., all its coefficients are 0.

Cantor's proof used an idea of Riemann: That much of the behavior of (1) can be inferred from studying its formal second integral, $\frac{1}{4} a_{0} x^{2}-$ $\sum_{n=1}^{\infty}\left(a_{n} \cos n x+b_{n}, \sin n x\right) / n^{2}$. For this proof see [7, p. 326], [1, pp. 14] or [3]. The idea of the present work is to use the first formal integral, $L(x):=\frac{1}{2} a_{0} x+\sum_{n=1}^{\infty}\left(a_{n} \sin n x-b_{n} \cos n x\right) / n$. ( $L$ is for Lebesgue.) Zygmund points out that the difficulty in using $L(x)$ is that $L(x)$ need not converge everywhere even if the series (1) does. (For example $\sum \sin n x / \log n$ converges everywhere but $-\sum \cos n 0 / n \log n$ diverges.) Nevertheless, here is a proof, dedicated to the would be extenders of Cantor's theorem, which uses $L$.

Proof. By the theorem of Cantor-Lebesgue ([7, p. 316], [1, Appendix 1] or [2]), $a_{n}$ and $b_{n}$ tend to 0 as $n$ tends to $\infty$ so that the coefficients of the series part of $L$ are $o(1 / n)$, whence the sum of their squares is finite. By the Riesz-Fisher Theorem, this series represents an $L^{2}$ function. ([7, p. 127]) A theorem of Rajchman and Zygmund says that at every point the symmetric approximate derivative of $L$ is equal to (the value of (1) which is) 0 . ([7, p. 324]) We may also assume that $L$ is approximately continuous at every

Received by the editors January 6, 1989.

1980 Mathematics Subject Classification (1985 Revision). Primary 42A63; Secondary 26A24. 
point of a $2 \pi$-periodic set $E$ of full Lebesgue measure, since every measurable function is approximately continuous a.e. ([6, Vol. 2, p. 257]). Since $L(x)$ is approximately continuous and has non-negative symmetric derivative on $E$, by a recent elementary but ingenious and difficult result of $\mathrm{C}$. Freiling and D. Rinne, $L$ is non-decreasing on $E$ ([4] and [5, Theorem 2]). Symmetrically $L$ is non-increasing on $E$, so that there is a constant $c$ with $L(x)=c$ for all $x$ in $E$. In other words, for all $x \in E$,

$$
-c+\sum_{n=1}^{\infty}\left(a_{n} \sin n x-b_{n} \cos n x\right) / n=-\frac{1}{2} a_{0} x .
$$

The left side of equation (2) represents an $L^{2}$ function and is therefore Abel summable a.e. to that function ([7, p. 90, Equation 3.9 and p. 80, Equation 1.33]). At each point of Abel summability Tauber's original Tauberian theorem (recall the coefficients are $o(1 / n))$ guarantees convergence $([7$, p. 81]). Fix one such point $x_{0}$ which is also in $E$. Since equation (2) holds at $x_{0}$ and at $x_{0}+2 \pi$ and the left side has the same value at both points, it follows that $a_{0}=0$. This means that the $L^{2}$ function represented by the left side of equation (2) is 0 a.e. Bessel's inequality ([7, p. 13, Equation 7.5]) gives that $(-c)^{2}+\sum\left(a_{n}^{2}+b_{n}^{2}\right) / n^{2} \leq$ 0 . The Theorem is proved.

Remark. The theorem of Freiling and Rinne which replaces the theorem of Schwarz and Cantor ([1, Appendix 2], [7, pp. 23 and 326], or [3]) that appears in the classical proof of the Theorem seems to avoid the maximum principle. However, Freiling and Rinne's present proof seems to require special properties of $\mathbf{R}^{1}$ that are not enjoyed by $\mathbf{R}^{n}$ for $n>1$.

\section{REFERENCES}

1. J. M. Ash, Uniqueness of representation by trigonometric series, Amer. Math. Monthly, (to appear).

2. G. Cantor, Über einen die trigonometrischen Reihen betreffenden Lehrsatz, Crelles J. für Math. 72 (1870) 130-138; also in Gesammelte Abhandlungen, Georg Olms, Hildesheim, 1962, 71-79.

3. __ Beweis, das eine für jeden reellen Wert von $x$ durch eine trigonometrische Reihe gegebene Funktion $f(x)$ sich nur auf eine einzige Weise in dieser Form darstellen lässt, Crelles J. für Math. 72 (1870) 139-142; also in Gesammelte Abhandlungen, Georg Olms, Hildesheim, 1962, 80-83.

4. C. Freiling and D. Rinne, A symmetric density property: monotonicity and the approximate symmetric derivative, Proc. of the Amer. Math. Soc., 104 (1988) 1098-1102.

5. $\ldots$, A symmetric density property for measurable sets, Real Analysis Exchange, 14 (1988-89) 203-209.

6. E. W. Hobson, The theory of functions of a real variable and the theory of Fourier's series, 2 vols., Dover Publications, New York, 1957, MR 19 \#1166.

7. A. Zygmund. Trigonometric series, vol. 1, 2nd rev. ed., Cambridge Univ. Press, New York, 1959, MR 21 \#6498.

Department of Mathematics, De Paul University, Chicago, Illinois 60614 